Tohoku J. Exp. Med., 2010, 221, 211-219

\title{
Contrast-Induced Nephropathy in Postmenopausal Women Undergoing Percutaneous Coronary Intervention for Acute Myocardial Infarction
}

\author{
Guizhou Ma, ${ }^{1}$ Danqing Yu, ${ }^{2}$ Zhixiong Cai, ${ }^{1}$ Chumin Ni, ${ }^{1}$ Ronghe Xu, ${ }^{1}$ Bin Lan, \\ Ping Chen ${ }^{1}$ and Zhidan Zhu ${ }^{1}$ \\ ${ }^{1}$ Department of Cardiology, Affiliated Shantou Hospital of Sun Yat-sen University, Shantou City, Guangdong \\ Province, PR China \\ ${ }^{2}$ Department of Coronary Care Unit, Guangdong Cardiovascular Institute, Guangdong Academy of Medical \\ Sciences, Guangdong Provincial People's Hospital, Guangzhou City, Guangdong Province, PR China
}

\begin{abstract}
Contrast-induced nephropathy $(\mathrm{CIN})$ is a complex syndrome of acute kidney injury induced by exposure to intravascular contrast media. CIN occurs frequently in patients undergoing urgent percutaneous coronary intervention ( $\mathrm{PCl}$ ) and is associated with poor outcomes, making it a major challenge faced by interventional cardiologists. It has been suggested that female sex is a risk factor for development of CIN following $\mathrm{PCl}$. However, no data exist in the literature concerning the risks of postmenopausal women with acute myocardial infarction (AMI) developing $\mathrm{CIN}$ after undergoing urgent $\mathrm{PCl}$. To explore the incidence, risk factors and in-hospital outcomes of CIN in this special population, we analyzed 69 postmenopausal women with $\mathrm{AMI}$ treated with urgent $\mathrm{PCl}$. CIN was defined as a relative increase of $>25 \%$ or an absolute increase of $\geq 0.5 \mathrm{mg} / \mathrm{dL}$ in serum creatinine concentration from the baseline value $72 \mathrm{~h}$ after exposure to contrast medium. We found 1) the incidence of CIN was $37.68 \%$; 2) patients with CIN had worse in-hospital outcomes, including longer hospital stay and more in-hospital adverse events; and 3) in multivariate logistic analysis, independent risk factors for CIN included a longer menopausal duration and the implantation of an intra-aortic balloon pump (an indirect indicator of hemodynamic instability). These results indicate that CIN is a frequent complication associated with worse in-hospital outcomes in postmenopausal women with AMI who are undergoing urgent $\mathrm{PCl}$, particularly those with longer menopausal duration and hemodynamic instability. It is therefore necessary to pay more attention to preventive strategies for renal protection in this special population.
\end{abstract}

Keywords: acute myocardial infarction; percutaneous coronary intervention; contrast-induced nephropathy; serum creatinine concentration; postmenopausal women

Tohoku J. Exp. Med., 2010, 221 (3), 211-219. C 2010 Tohoku University Medical Press

Contrast-induced nephropathy (CIN) is a complex syndrome of acute kidney injury that follows exposure to intravascular contrast media. It is the third leading cause of hospital-acquired acute renal failure (Maeder et al. 2004). It occurs frequently in patients undergoing urgent percutaneous coronary intervention (PCI) for acute myocardial infarction (AMI), with incidence rates reaching 10.82\% 28.00\% (Marenzi et al. 2004, 2009; Valente et al. 2006; Bouzas-Mosquera et al. 2007; Senoo et al. 2010). Its development is associated with poor outcomes (McCullough et al. 1997; Gruberg et al. 2000; Rihal et al. 2002; Marenzi et al. 2004, 2009; Valente et al. 2006; Bouzas-Mosquera et al. 2007; Shema et al. 2009; Wickenbrock et al. 2009), including prolonged hospitalization, increased in-hospital mortali- ty rates and increased long-term adverse events. Therefore, CIN has become a major challenge faced by interventional cardiologists in the era of routine PCI.

Postmenopausal women are a special population that plays a distinctive role in coronary heart disease. The incidence of coronary heart disease increases significantly after menopause in women (Gordon et al. 1978; Colditz et al. 1987). Therefore, postmenopausal women account for a considerable percentage of the population who are treated in the urgent care setting with PCI. Studies have shown that female sex is independently associated with development of CIN in patients undergoing PCI (Senoo et al. 2010); moreover, the risk of developing CIN for women has been determined to be four times that for men (Mueller et al. 2002).

Received January 13, 2010; revision accepted for publication May 24, 2010. doi:10.1620/tjem.221.211

Correspondence: Danqing Yu, Department of Coronary Care Unit, Guangdong Cardiovascular Institute, Guangdong Academy of

Medical Sciences, Guangdong Provincial People's Hospital, 510080, Guangzhou City, Guangdong Province, PR China.

e-mail: gdydq@medmail.com.cn 
However, there are still no data available on CIN occurrence in postmenopausal women undergoing urgent PCI for AMI. Thus, the aim of our study was to explore the incidence, risk factors, and in-hospital outcomes of CIN in this special population.

\section{Materials and Methods}

Study population

Between May 1, 2008 and October 31, 2009, we enrolled all consecutive postmenopausal (defined as menopause occurring more than one year prior) female patients admitted to our coronary care unit (CCU) for AMI who were treated with urgent PCI. The study procedure was carried out according to the protocol approved by the Ethics Committee of the Affiliated Shantou Hospital of Sun Yat-sen University, and written informed consent was obtained from all patients.

According to the ACC/AHA guidelines for primary PCI in AMI, postmenopausal female patients were included if they presented within $12 \mathrm{~h}$ (18 h for AMI complicated by cardiogenic shock) from the onset of symptoms. The symptoms were as follows: characteristic chest pain lasting for at least $30 \mathrm{~min}$ with an electrocardiographic ST-segment elevation of at least $0.2 \mathrm{mV}$ in two or more contiguous leads; a left bundle branch block; and, a demonstration of elevated serum markers, including Troponin I, Troponin T or CK-MB but without ST-segment elevation. Patients were excluded if they fulfilled one of the following criteria: 1) the coronary anatomy was not suitable for PCI; 2) an emergency bypass grafting was required; 3) death occurred within the first $48 \mathrm{~h}$; and 4) PCI treatment was refused.

\section{Study protocol}

Urgent PCI

Urgent PCI was performed by a 24-h on-call emergency team, composed of interventional cardiologists. In most cases, the femoral approach was used; but when clinically appropriate, the radial approach was used.

Before PCI: Patients received oral loading doses (300 mg each) of aspirin and clopidogrel in the emergency department or in the cardiac catheterization room.

During PCI: A bolus injection of unfractionated heparin $(7,000$ $\mathrm{U})$ was administered at the beginning of the PCI procedure, followed by additional boluses during the procedure according to the condition of the patient and the experience of the interventional cardiologists. All patients received percutaneous transluminal coronary angioplasty and/or implantation of stents according to the condition of the coronary artery. Iopamidol-370 (Bracoo, Milan, Italy) and Iopromide (Schering, Berlin, Germany) were the non-ionic, monomeric, hypoosmolar contrast media employed. Each patient was randomly assigned to receive one of the two contrast media.

After PCI: Physiologic $(0.9 \%)$ saline was also administered in travenously for $12 \mathrm{~h}$ after PCI according to the patients' cardiac function. All patients received aspirin and clopidogrel at standard dosages as part of the post-stenting antithrombotic treatment. Other conventional therapeutic measures included administration of angiotensinconverting enzyme inhibitors (ACEI), angiotensin II receptor blockers (ARB), beta-adrenergic blockers, platelet glycoprotein IIb/IIIa receptor inhibitors (tirofiban), diuretics, inotropic drugs or the implantation of an intra-aortic balloon pump (IABP, a mechanical device used to decrease myocardial oxygen demand and increase cardiac output).
The application of these drugs and the IABP was carried out at the discretion of the interventional and CCU cardiologists, and the drugs were administered according to the standard clinical protocols and international guidelines (Van de Werf et al. 2003; Gluckman et al. 2005).

\section{Testing indexes}

Serum creatinine concentration ( $\mathrm{SCr}$ ) was measured upon hospital admission (just before primary PCI) and then daily for three days following PCI. Serum lipid profile, uric acid and serum N-terminal pro-brain natriuretic peptide (NT-proBNP, a cardiac natriuretic peptide used as a clinical hormonal marker in cardiac dysfunction) were also measured within $24 \mathrm{~h}$ after PCI. Transthoracic echocardiography was performed within $24 \mathrm{~h}$ from admission to evaluate the left ventricular ejection fraction (LVEF) in all patients, and the results were recorded and interpreted by experienced experts.

\section{Record of in-hospital adverse events}

During hospitalization, the following adverse events were considered: acute heart failure, major ventricular arrhythmias (ventricular tachycardia and ventricular fibrillation), intra-aortic balloon pump implantation, renal replacement therapy, nosocomial infection and death.

\section{Definitions}

PCI was defined as urgent when it was used to treat AMI within $12 \mathrm{~h}$ ( $18 \mathrm{~h}$ for AMI complicated by cardiogenic shock) from the onset of symptoms.

CIN was defined as a relative increase of $>25 \%$ or an absolute increase of $\geq 0.5 \mathrm{mg} / \mathrm{dL}$ in $\mathrm{SCr}$ from the baseline value $72 \mathrm{~h}$ after exposure to the contrast medium (Morcos et al. 1999).

Creatinine clearance was calculated by applying the CockcroftGault formula to the SCr (Cockcroft and Gault 1976): [(140 - age) $\times$ $\mathrm{Wt}(\mathrm{kg}) \times 0.85] /$ serum creatinine $(\mathrm{mg} / \mathrm{dL})$.

Maximum contrast dose (MCD) was calculated by applying the following formula proposed by Cigarroa and colleagues (Cigarroa et al. 1989): $\mathrm{MCD}(\mathrm{mL})=(5 \times$ body weight $[\mathrm{kg}]) /$ serum creatinine $(\mathrm{mg} / \mathrm{dL})$.

The contrast ratio was calculated by dividing the contrast amount administered by the calculated MCD (Marenzi et al. 2009).

\section{Statistical analysis}

Statistical analysis was performed using SPSS (version 16.0; Chicago, IL, USA). Continuous variables following a symmetric distribution were presented as means and standard deviations $(\overline{\mathrm{X}}+S D)$ and were compared using the two independent samples $t$-test. Variables following an asymmetric distribution were presented as medians and interquartile ranges $[\mathrm{M}(\mathrm{P} 25, \mathrm{P} 75)]$ and were compared using the Kruskal-Wallis nonparametric test. Categorical variables were described as frequencies and analyzed by chi-square tests and Fischer's exact tests. A two-step analysis was used to identify the independent factors for CIN. First, a univariate logistic analysis was performed to select the risk factors for CIN. Second, a stepwise multivariate logistic model was developed by consideration of variables that were clinically relevant to the univariate logistic analysis. Results were presented as odds ratios $(O R)$ with $95 \%$ confidence intervals. A $P$ value $\leq 0.05$ was considered statistically significant. 


\section{Results}

Clinical characteristics of the entire study population

Between May 1, 2008 and October 31, 2009, 79 consecutive patients were enrolled in our study. However, six patients died before PCI, two were not suitable for PCI because of coronary anatomy (one patient died within $24 \mathrm{~h}$ after coronary angiography [CAG] and the other received emergency bypass grafting), and two patients died within 48 $\mathrm{h}$ after PCI. Therefore, 69 consecutive patients were analyzed in our study (Fig. 1).

All patients were postmenopausal women. Of them, 66 patients had ST-elevating myocardial infarction (STEMI) and three had non-ST-elevating myocardial infarction (NSTEMI). Of them, 34 patients received Iopamidol-370 and 35 received Iopromide. Their mean age was $68.78 \pm$ 9.18 years, their mean menopausal age was $50.29 \pm 2.03$ years, and their mean menopausal duration was $18.28 \pm 9.55$ years. The mean baseline creatinine clearance of the study population was $54.70 \pm 25.11 \mathrm{ml} / \mathrm{min}$, and 48 patients had a moderately impaired renal function $(<60 \mathrm{ml} / \mathrm{min})$, accounting for approximately $69.57 \%$ of the patients. A total of 33 (47.83\%) patients were diagnosed with three-vessel disease (a severe coronary artery disease involving the left anterior descending, the left circumflex and the right coronary artery). The culprit vessels (the infarct-related coronary arteries) were the left anterior descending (35 cases, 50.72\%), the left circumflex (13 cases, 18.84\%), and the right coronary artery (21 cases, $30.43 \%$ ).

In our study population, 26 patients developed CIN after urgent PCI and the overall incidence reached $37.68 \%$.

\section{Baseline clinical and procedural characteristics in patients with and without CIN}

The baseline clinical and procedural characteristics of patients with and without CIN after urgent PCI are shown in Table 1. Patients with CIN were older; they typically had a longer menopausal duration (usually longer than 10 years) and were older than 65 years. Moreover, patients with CIN more frequently presented with a history of hypertension and renal insufficiency and typically had 1) a culprit vessel of the left anterior descending coronary artery, 2) a higher Killip's class ( $\geq 3), 3$ ) a lower LVEF $(\leq 45 \%)$, 4) higher serum uric acid and NT-proBNP levels, and 5) a higher contrast ratio $(\geq 1)$. In addition, a trend towards a higher per-

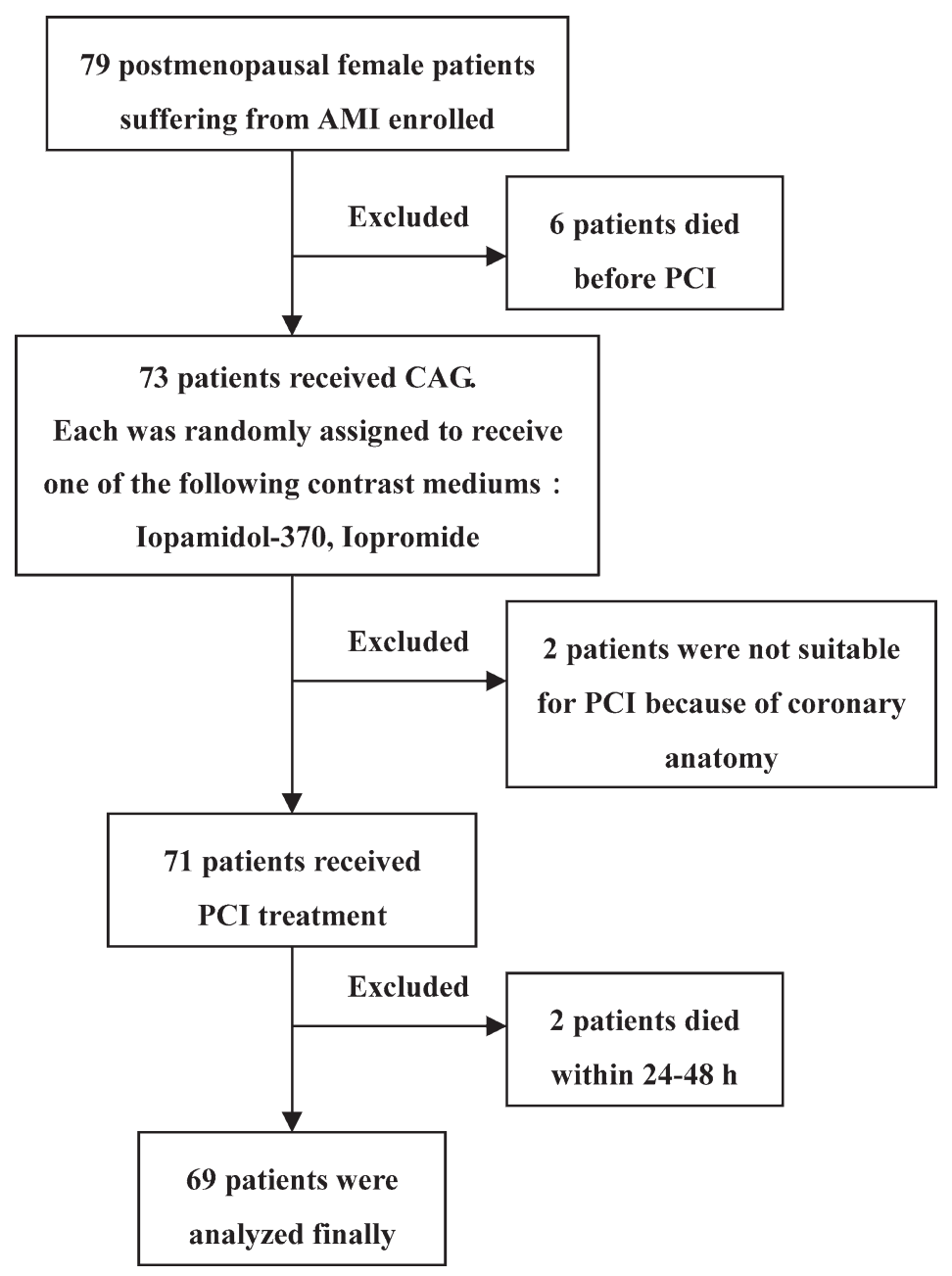

Fig. 1. Flow chart of the patient selection process. 
Table 1. Baseline clinical and procedural characteristics of the study patients.

\begin{tabular}{|c|c|c|c|}
\hline & no-CIN & $\mathrm{CIN}$ & \multirow{2}{*}{$P$ Value } \\
\hline & $n=43(62.32 \%)$ & $n=26(37.68 \%)$ & \\
\hline \multicolumn{4}{|l|}{ Demographics } \\
\hline Mean age, years & $66.81 \pm 9.32$ & $72.04 \pm 8.08$ & 0.021 \\
\hline Mean menopausal duration, years & $16.23 \pm 9.52$ & $21.65 \pm 8.76$ & 0.021 \\
\hline Age $\geq 65$ years & $26(60.47 \%)$ & $22(84.62 \%)$ & 0.035 \\
\hline Menopausal duration $\geq 10$ years & $27(62.79 \%)$ & $24(92.31 \%)$ & 0.007 \\
\hline \multicolumn{4}{|l|}{ Past history } \\
\hline Hypertension & $23(53.49 \%)$ & $21(80.77 \%)$ & 0.022 \\
\hline Diabetes mellitus & $8(18.60 \%)$ & $7(26.92 \%)$ & 0.417 \\
\hline Renal insufficiency & $5(11.63 \%)$ & $9(34.62 \%)$ & 0.021 \\
\hline Previous myocardial infarction / PCI & $0(0.00 \%)$ & $0(0.00 \%)$ & - \\
\hline \multicolumn{4}{|l|}{ Laboratory parameters } \\
\hline Serum total cholesterol, $\mathrm{mmol} / \mathrm{L}$ & $5.04 \pm 1.04$ & $5.25 \pm 1.30$ & 0.672 \\
\hline Serum uric acid, $\mathrm{mmol} / \mathrm{L}$ & $286.58 \pm 68.57$ & $402.00 \pm 118.60$ & $<0.001$ \\
\hline Serum NT-proBNP, pg/ml & $\begin{array}{c}3505.00 \\
(2213.00,3505.00)\end{array}$ & $\begin{array}{c}10058.00 \\
(4107.00,10373.00)\end{array}$ & $<0.001$ \\
\hline Creatinine clearance, $\mathrm{ml} / \mathrm{min}$ & $\begin{array}{c}52.71 \\
(41.34,72.27)\end{array}$ & $\begin{array}{c}45.83 \\
(29.63,63.94)\end{array}$ & 0.162 \\
\hline Fasting blood glucose, $\mathrm{mmol} / \mathrm{L}$ & $5.93(5.53,7.58)$ & $6.92(5.90,7.96)$ & 0.096 \\
\hline \multicolumn{4}{|l|}{ Cardiovascular characteristics } \\
\hline STEMI & $41(95.35 \%)$ & $25(96.15 \%)$ & $1.000^{*}$ \\
\hline NSTEMI & $2(4.65 \%)$ & $1(3.85 \%)$ & $1.000^{*}$ \\
\hline Mean LVEF, \% & $55.51 \pm 9.76$ & $47.12 \pm 9.81$ & 0.001 \\
\hline $\mathrm{LVEF} \leq 45 \%$ & $6(13.95 \%)$ & $10(38.46 \%)$ & 0.019 \\
\hline Killip's class $\geq 3$ & $3(6.98 \%)$ & $11(42.31 \%)$ & $<0.001$ \\
\hline Three-vessel disease & $21(48.84 \%)$ & $12(46.15 \%)$ & 0.829 \\
\hline Left main coronary artery disease & $1(2.33 \%)$ & $3(11.54 \%)$ & $0.147^{*}$ \\
\hline \multicolumn{4}{|l|}{ Culprit vessels } \\
\hline Left anterior descending & $17(39.53 \%)$ & $18(69.23 \%)$ & 0.017 \\
\hline Left circumflex & $11(25.58 \%)$ & $2(7.69 \%)$ & 0.066 \\
\hline Right coronary artery & $15(34.88 \%)$ & $6(23.08 \%)$ & 0.302 \\
\hline \multicolumn{4}{|l|}{ Combined medications } \\
\hline Diuretic agents & $8(18.60 \%)$ & $20(76.92 \%)$ & $<0.001$ \\
\hline ACEI / ARB & $40(93.02 \%)$ & $24(92.31 \%)$ & $1.000^{*}$ \\
\hline \multicolumn{4}{|l|}{ Contrast media } \\
\hline Mean contrast volume, $\mathrm{mL}$ & $160.47 \pm 45.72$ & $180.77 \pm 53.06$ & 0.158 \\
\hline Iopamidol-370 & $23(53.49 \%)$ & $11(42.31 \%)$ & 0.368 \\
\hline Iopromide & $20(46.51 \%)$ & $15(57.69 \%)$ & 0.368 \\
\hline Contrast ratio $\geq 1$ & $2(4.65 \%)$ & $6(23.08 \%)$ & $0.046^{*}$ \\
\hline
\end{tabular}

*By Fisher's exact test. Culprit vessels refer to the infarct-related coronary arteries. CIN, contrast-induced nephropathy; PCI, percutaneous coronary intervention; STEMI, ST-elevating myocardial infarction; NSTEMI, non-ST-elevating myocardial infarction; LVEF, left ventricular ejection fraction; ACEI, angiotensin-converting enzyme inhibitors; ARB, angiotensin II receptor blockers.

centage of treatment with diuretic agents, but not ACEI or $\mathrm{ARB}$, was observed in patients with CIN.

There was no significant difference in the presence of diabetes mellitus, the mean contrast volume or creatinine clearance between patients with and without CIN. The differences in cardiovascular characteristics (including STEMI, NSTEMI, three-vessel disease and left main coronary artery disease), serum total cholesterol and fasting blood glucose between patients with and without CIN were also not statistically significant.

\section{Risk factors for CIN}

In univariate logistic analysis (Table 2), risk factors for the development of CIN after urgent PCI were as follows: 
Table 2. Risk factors for CIN by univariate logistic analysis.

\begin{tabular}{lccc}
\hline & OR & $95 \%$ CI & P value \\
\hline Demographics & & & \\
Age & 1.070 & $1.008 \sim 1.137$ & 0.026 \\
Menopausal duration & 1.066 & $1.008 \sim 1.128$ & 0.026 \\
Past history & & & 0.027 \\
Hypertension & 3.652 & $1.163 \sim 11.473$ & 0.419 \\
Diabetes mellitus & 1.612 & $0.506 \sim 5.131$ & 0.027 \\
Renal insufficiency & 4.024 & $1.172 \sim 13.817$ & $<0.001$ \\
Laboratory parameters & & & $<0.001$ \\
Serum uric acid & 1.014 & $1.007 \sim 1.022$ & 0.385 \\
Serum NT-proBNP & 1.000 & $1.000 \sim 1.001$ & \\
Creatinine clearance & 0.991 & $0.971 \sim 1.012$ & 0.024 \\
Cardiovascular characteristics & & & 0.002 \\
LVEF $\leq 45 \%$ & 3.854 & $1.197 \sim 12.415$ & \\
Killip's class $\geq 3$ & 9.778 & $2.392 \sim 39.961$ & $<0.001$ \\
Combined therapies & & & 0.912 \\
Diuretic agents & 14.583 & $4.425 \sim 48.061$ & 0.004 \\
ACEI/ARB & 0.900 & $0.140 \sim 5.778$ & 0.253 \\
IABP & 10.853 & $2.120 \sim 55.568$ & 0.035 \\
Contrast media & & & \\
Contrast medium volume & 1.006 & $0.996 \sim 1.016$ & \\
Contrast ratio $\geq 1$ & 6.150 & $1.138 \sim 33.238$ & \\
\hline
\end{tabular}

CIN, contrast-induced nephropathy; OR, odds ratio; CI, confidence interval; LVEF, left ventricular ejection fraction; ACEI, angiotensin-converting enzyme inhibitors; ARB, angiotensin II receptor blockers; IABP, intra-aortic balloon pump.

Table 3. Independent risk factors for CIN by multivariate logistic analysis.

\begin{tabular}{crcc}
\hline & OR & 95\%CI & P value \\
\hline Menopausal duration & 1.084 & $1.016 \sim 1.155$ & 0.013 \\
IABP & 14.724 & $2.597 \sim 83.488$ & 0.002 \\
\hline
\end{tabular}

CIN, contrast-induced nephropathy; OR, odds ratio; CI, confidence interval; IABP, intra-aortic balloon pump. Variables in the multivariate logistic analysis: menopausal duration, hypertension, diabetes mellitus, pre-renal insufficiency, IABP, contrast ratio $\geq 1$.

age; menopausal duration; hypertension; pre-renal insufficiency; serum uric acid; serum NT-proBNP; LVEF $\leq 45 \%$; Killip's class $\geq 3$; IABP; treatment with diuretic agents; and, contrast ratio $\geq 1$.

In multivariate logistic analysis (Table 3 ), because of the limited number of enrolled patients, we had to develop variables that were more likely to be related to CIN from a clinical standpoint according to the univariate logistic analysis. Variables included menopausal duration, hypertension, diabetes mellitus, pre-renal insufficiency, IABP, and contrast ratio $\geq 1$. Finally, only menopausal duration and IABP were shown to be independent risk factors for CIN.

\section{The relationship between CIN and in-hospital outcomes}

Patients with CIN experienced worse in-hospital clinical outcomes (Table 4 and Fig. 2). All in-hospital adverse events occurred more frequently in patients with CIN. Of those patients with CIN, two suffered from nosocomial infection, two from acute heart failure and two from ventricular fibrillation, whereas none of the patients without CIN exhibited these symptoms. The difference in the total incidence of the aforementioned adverse clinical events between patients with and without CIN was statistically significant $(P=0.002)$. Patients with CIN had a higher percentage of IABP implantation and renal replacement therapy. However, there was no significant difference found in the in-hospital mortality rate between the two groups in our study. The average length of hospital stay in patients with CIN was almost twice that of patients without CIN $(P<0.001)$. 
Table 4. In-hospital adverse events.

\begin{tabular}{lccc}
\hline & no-CIN & CIN & P Value \\
\cline { 2 - 3 } & $n=43(62.32 \%)$ & $n=26(37.68 \%)$ & $0.002 *$ \\
Nosocomial infection, acute heart failure and ventricular fibrillation & $0(0.00 \%)$ & $6(23.08 \%)$ & 0.002 \\
Requiring IABP & $2(4.65 \%)$ & $9(34.62 \%)$ & $0.050 *$ \\
Requiring renal replacement therapy & $0(0.00 \%)$ & $3(11.54 \%)$ & $2(7.69 \%)$ \\
In-hospital death & $0(0.00 \%)$ & $0.139 *$ \\
\hline
\end{tabular}

* By Fisher's exact test.

CIN, contrast-induced nephropathy; IABP, intra-aortic balloon pump.

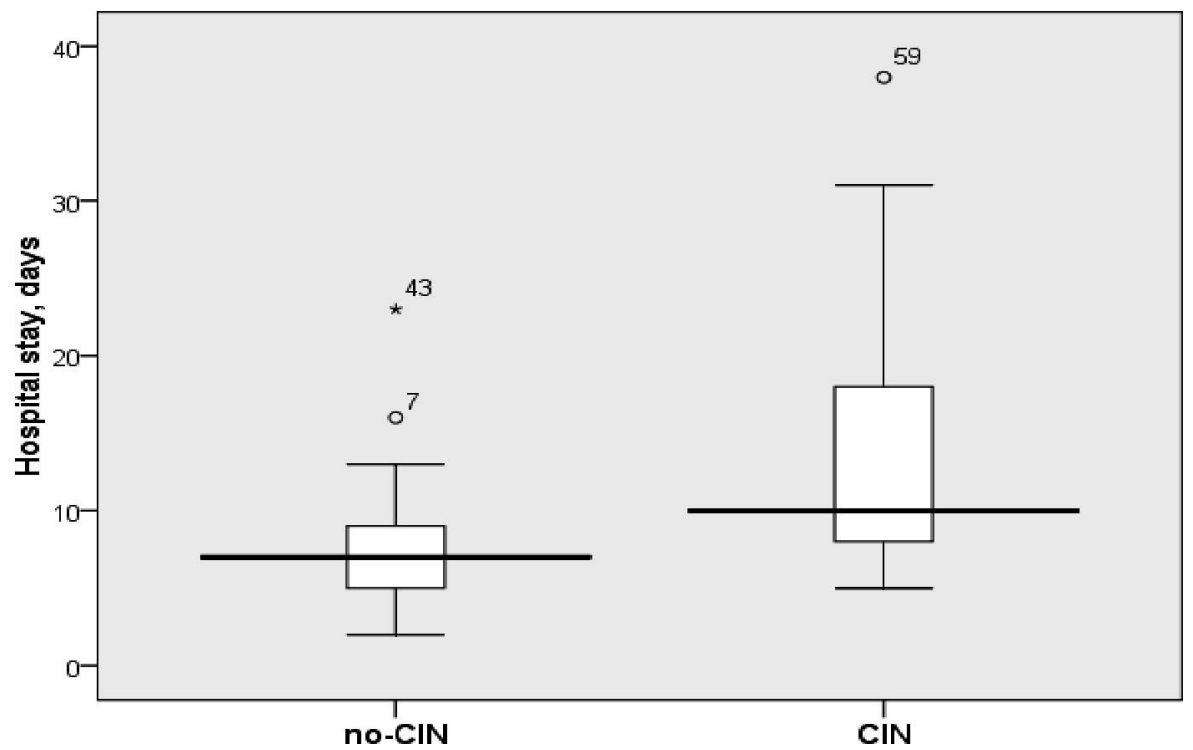

Fig. 2. The average length of hospital stay.

${ }^{\circ} 7$ : the $7^{\text {th }}$ patient stayed at the hospital for 16 days; $* 43$ : the $43^{\text {th }}$ patient stayed at the hospital for 23 days; ${ }^{\circ} 59$ : the $59^{\text {th }}$ patient stayed at the hospital for 38 days.

\section{Discussion}

To our knowledge, this study is the first to reveal information regarding CIN in postmenopausal women with AMI undergoing urgent PCI. Our findings demonstrate that CIN is a frequent complication associated with worse in-hospital outcomes in this special population, particularly in those patients with longer menopausal duration and hemodynamic instability.

\section{Incidence of CIN in postmenopausal women undergoing urgent PCI for AMI}

Previous studies have shown that the incidence of CIN in the general patient population with AMI undergoing urgent PCI was between $10.82 \%$ and $28.00 \%$ (Marenzi et al. 2004, 2009; Valente et al. 2006; Bouzas-Mosquera et al. 2007; Senoo et al. 2010).

In our study, we focused our evaluation on the development of CIN during AMI in postmenopausal women. We found that the overall incidence of CIN was $37.68 \%$, which was significantly higher than that reported in previous studies. We believe this finding to be attributable to the follow- ing factors.

First, the different definition of CIN may be an important factor. Some researchers have reported that the incidence of CIN was sensitive to the definition used (Toprak and Mueller 2005; Jabara et al. 2009). The incidence of CIN was lower when the definition of an absolute increase in $\mathrm{SCr}>0.5 \mathrm{mg} / \mathrm{dL}$ was used (Toprak and Mueller 2005). In previous studies (Marenzi et al. 2004; Valente et al. 2006; Bouzas-Mosquera et al. 2007), a rise of $\geq 0.5 \mathrm{mg} / \mathrm{dL}$ or $>0.5$ $\mathrm{mg} / \mathrm{dL}$ in $\mathrm{SCr}$ was used to define CIN. However, in our study, we used a relative increase of $>25 \%$ or an absolute increase of $\geq 0.5 \mathrm{mg} / \mathrm{dL}$ in $\mathrm{SCr}$ from the baseline value to define CIN, which was different from that of previous studies.

Second, the different study population may have affected the different incidence observed. Compared to the general population, postmenopausal women were older and often presented with other risk factors, such as diabetes mellitus, hypertension and atherosclerosis. Renal atherosclerosis was common in our postmenopausal patient population, and renal function was vulnerable in these patients. Previous studies have shown that the incidence of CIN was 
significantly higher in women than in men (Mueller et al. 2002, 2004; Iakovou et al. 2003). Therefore, our special study population may explain the high incidence observed.

Third, the different baseline renal function was an important factor that should not be ignored. A great number of patients (up to $69.57 \%$ ) showed a reduced baseline renal function $(<60 \mathrm{ml} / \mathrm{min})$ in our study, which was another important reason for the high incidence of CIN. According to the study by Marenzi et al. (2004), the incidence of CIN in patients with a creatinine clearance of $<60 \mathrm{ml} / \mathrm{min}$ reached 40\%. Mueller et al. (2004) have also reported that the significantly higher incidence of CIN after PCI in women may be related to their lower baseline glomerular filtration rate.

Fourth, the racial difference may have contributed to the high incidence rate. Some researchers have reported a higher incidence of CIN in non-whites and African Americans (Lindsay et al. 2003; Dangas et al. 2005). However, this remains to be verified in a Chinese population.

Finally, other potential reasons for the high incidence of CIN included the direct toxicity of the contrast media (Humes et al. 1987), the electrical and hemodynamic instability, and the lack of time to undergo adequate prophylaxis in these patients (Wright et al. 2002; Marenzi et al. 2003; Maeder et al. 2004).

\section{Clinical features of CIN in postmenopausal women undergoing urgent PCI for AMI}

Previous studies have shown that patients with CIN were older in the general patient population (Valente et al. 2006; Bouzas-Mosquera et al. 2007; Wickenbrock et al. 2009). Although our study population was strictly composed of postmenopausal women, we also showed that patients with CIN were older, and typically 1) had a longer menopausal duration (usually longer than 10 years) and 2) were older than 65 years. Generally speaking, the older the patient is the longer menopausal duration she has. An older age is, in essence, the same as a longer menopausal duration. Therefore, our study confirmed that patients with CIN were more frequently of an older age.

In our study, patients with CIN more frequently presented with 1) a history of hypertension and renal insufficiency, 2) a culprit vessel of the left anterior descending coronary artery, 3) a higher Killip's class, 4) a lower LVEF, 5) higher serum uric acid and NT-proBNP levels, 6) a higher contrast ratio ( $\geq 1)$, and 7 ) a higher percentage of treatment with diuretic agents. These clinical features are partially consistent with previous studies (Weinstein et al. 1992; Solomon et al. 1994; Freeman et al. 2002; BouzasMosquera et al. 2007; Marenzi et al. 2009). Usually, more contrast media was used to perform PCI in the left anterior descending artery than in the right coronary artery (Senoo et al. 2010). This might explain why patients with CIN presented more frequently with a culprit vessel of the left anterior descending coronary artery (Senoo et al. 2010). Higher
Killip's class, lower LVEF, and high serum NT-proBNP have been associated with congestive heart failure, which is known to occur more frequently in patients with CIN (Rihal et al. 2002). Increased serum uric acid was also common in patients with CIN, and it may represent a true risk factor for acute renal failure (Ejaz et al. 2007). The higher contrast ratio $(\geq 1)$ meant that the contrast medium volume exceeded the MCD threshold and might lead to the deterioration of renal function (Freeman et al. 2002), which could explain why patients with CIN more frequently present with a higher contrast ratio.

Independent predictive factors for CIN in postmenopausal women undergoing urgent PCI for AMI

The use of IABP (an indirect indicator of hemodynamic instability) was reported to be a strong independent predictive factor for CIN (Marenzi et al. 2004; Mehran et al. 2004). Our study has confirmed this result, strongly suggesting that the development of CIN after urgent PCI was highly correlated with hemodynamic instability.

The second independent predictive factor for CIN was menopausal duration, which was the defining characteristic of our study population. Because a longer menopausal duration is relatively the same as an older age, this finding could be considered as consistent with that reported by Valente et al. (2006).

Although pre-renal insufficiency and diabetes mellitus were considered to be two independent risk factors for CIN (Bouzas-Mosquera et al. 2007), their presence did not confer increased risks of CIN in our study. The similar result was reported by Valente et al. (2006). It is possible, however, that the different study populations in our study can explain the discrepant findings.

Some of the previous studies indicated contrast medium volume as another risk factor for CIN (Marenzi et al. 2004, 2009). Because the contrast ratio, but not contrast medium volume, was based on the individual difference of the patients, it could reflect influences of patient age, weight and renal function. Thus, contrast ratio was considered as a potential risk factor and screened in our study. However, contrast ratio was not identified by multivariate analysis as an independent factor for CIN in our study.

\section{The relationship between CIN and in-hospital outcomes} Many studies (Marenzi et al. 2004, 2009; Valente et al. 2006; Bouzas-Mosquera et al. 2007; Wickenbrock et al. 2009) have demonstrated that patients with CIN had worse in-hospital outcomes, including a more prolonged hospital stay, more complicated clinical course and significantly higher in-hospital mortality rates. In our study, patients with CIN also experienced a longer hospital stay and more clinical complications, including nosocomial infection, acute heart failure and ventricular fibrillation. The in-hospital mortality rate for postmenopausal patients with CIN was only $7.69 \%$, which is lower than the $9.52 \%$ to $31.00 \%$ reported in most previous studies (Marenzi et al. 2004, 
2009; Valente et al. 2006; Bouzas-Mosquera et al. 2007; Wickenbrock et al. 2009). This discrepancy may be related to the exclusion criteria used in our study (among those patients who were excluded, six died before PCI, one died within $24 \mathrm{~h}$ after CAG, and two died within $48 \mathrm{~h}$ after PCI) and the distinct study population. Although no statistical significance was obtained in the consideration of in-hospital mortality rate between patients with and without CIN in our study, the risk increased in patients with CIN compared to those without $(7.69 \%$ vs. $0.00 \%$, respectively). It is possible that the small study population made it difficult to draw a statistically significant conclusion.

\section{Conclusions}

Our study has confirmed that $\mathrm{CIN}$ is a frequent complication in patients undergoing PCI for AMI. In addition, we demonstrated that the incidence of CIN is quite high in postmenopausal women, particularly in those with longer menopausal duration and hemodynamic instability. In this special population, patients who develop CIN have worse in-hospital outcomes, including a longer hospital stay and more in-hospital adverse events. Thus, more attention should be given to preventive strategies for renal protection in postmenopausal women undergoing urgent PCI for AMI.

\section{Study limitations}

Our study has some limitations. First, the number of enrolled patients was small. As a rule of thumb, the recommended minimum observation-to-predictor ratio is 10:1 in multivariate statistics. Thus, we had to develop variables that were more likely to be related to CIN from a clinical standpoint according to the univariate logistic analysis. Second, our data came from a single center. Finally, there was no follow-up performed after discharge. Our findings should, therefore, be confirmed in a larger multicenter trial, and include follow-up after discharge.

\section{Acknowledgments}

The authors wish to thank the journal editor and reviewers for their valuable input, which helped to improve the report. This study was supported in part by the Natural Science Foundation of Guangdong Province, PR China (Grant Number: 07023131).

\section{References}

Bouzas-Mosquera, A., Vázquez-Rodríguez, J.M., Calviño-Santos, R., Peteiro-Vázquez, J., Flores-Ríos, X., Marzoa-Rivas, R., Piñón-Esteban, P., Aldama-López, G., Salgado-Fernández, J., Vázquez-González, N. \& Castro-Beiras, A. (2007) Contrastinduced nephropathy and acute renal failure following emergent cardiac catheterization: incidence, risk factors and prognosis. Rev. Esp. Cardiol., 60, 1026-1034.

Cigarroa, R.G., Lange, R.A., Williams, R.H. \& Hillis, L.D. (1989) Dosing of contrast material to prevent contrast nephropathy in patients with renal disease. Am. J. Med., 86, 649-652.

Cockcroft, D.W. \& Gault, M.H. (1976) Prediction of creatinine clearance from serum creatinine. Nephron, 16, 31-41.

Colditz, G.A., Willett, W.C., Stampfer, M.J., Rosner, B., Speizer, F.E. \& Hennekens, C.H. (1987) Menopause and the risk of coronary heart disease in women. N. Engl. J. Med., 316, 1105-1110.

Dangas, G., Iakovou, I., Nikolsky, E., Aymong, E.D., Mintz, G.S., Kipshidze, N.N., Lansky, A.J., Moussa, I., Stone, G.W., Moses, J.W., Leon, M.B. \& Mehran, R. (2005) Contrastinduced nephropathy after percutaneous coronary interventions in relation to chronic kidney disease and hemodynamic variables. Am. J. Cardiol., 95, 13-19.

Ejaz, A.A., Mu, W., Kang, D.H., Roncal, C., Sautin, Y.Y., Henderson, G., Tabah-Fisch, I., Keller, B., Beaver, T.M., Nakagawa, T. \& Johnson, R.J. (2007) Could uric acid have a role in acute renal failure? Clin. J. Am. Soc. Nephrol., 2, 16-21.

Freeman, R.V., O’Donnell, M., Share, D., Meengs, W.L., KlineRogers, E., Clark, V.L., DeFranco, A.C., Eagle, K.A., McGinnity, J.G., Patel, K., Maxwell-Eward, A., Bondie, D. \& Moscucci, M.; Blue Cross-Blue Shield of Michigan Cardiovascular Consortium (BMC2). (2002) Nephropathy requiring dialysis after percutaneous coronary intervention and the critical role of an adjusted contrast dose. Am. J. Cardiol., 90, 1068-1073.

Gluckman, T.J., Sachdev, M., Schulman, S.P. \& Blumenthal, R.S. (2005) A simplified approach to the management of non-STsegment elevation acute coronary syndromes. JAMA, 293, 349-357.

Gordon, T., Kannel, W.B., Hjortland, M.C. \& McNamara, P.M. (1978) Menopause and coronary heart disease. The Framingham Study. Ann. Intern. Med., 89, 157-161.

Gruberg, L., Mintz, G.S., Mehran, R., Dangas, G., Lansky, A.J., Kent, K.M., Pichard, A.D., Satler, L.F. \& Leon, M.B. (2000) The prognostic implications of further renal function deterioration within 48 hours of interventional coronary procedures in patients with pre-existent chronic renal insufficiency. $J$. Am. Coll. Cardiol., 36, 1542-1548.

Humes, H.D., Hunt, D.A. \& White, M.D. (1987) Direct toxic effect of the radiocontrast agent diatrizoate on renal proximal tubule cells. Am. J. Physiol., 252, F246-255.

Iakovou, I., Dangas, G., Mehran, R., Lansky, A.J., Ashby, D.T., Fahy, M., Mintz, G.S., Kent, K.M., Pichard, A.D., Satler, L.F., Stone, G.W. \& Leon, M.B. (2003) Impact of gender on the incidence and outcome of contrast-induced nephropathy after percutaneous coronary intervention. J. Invasive Cardiol., 15, $18-22$.

Jabara, R., Gadesam, R.R., Pendyala, L.K., Knopf, W.D., Chronos, N., Chen, J.P., Viel, K., King, S.B. 3rd \& Manoukian, S.V. (2009) Impact of the definition utilized on the rate of contrastinduced nephropathy in percutaneous coronary intervention. Am. J. Cardiol., 103, 1657-1662.

Lindsay, J., Apple, S., Pinnow, E.E., Gevorkian, N., Gruberg, L., Satler, L.F., Pichard, A.D., Kent, K.M., Suddath, W. \& Waksman, R. (2003) Percutaneous coronary interventionassociated nephropathy foreshadows increased risk of late adverse events in patients with normal baseline serum creatinine. Catheter Cardiovasc. Interv., 59, 338-343.

Maeder, M., Klein, M., Fehr, T. \& Rickli, H. (2004) Contrast nephropathy: review focusing on prevention. J. Am. Coll. Cardiol., 44, 1763-1771.

Marenzi, G., Assanelli, E., Campodonico, J., Lauri, G., Marana, I., De Metrio, M., Moltrasio, M., Grazi, M., Rubino, M., Veglia, F., Fabbiocchi, F. \& Bartorelli, A.L. (2009) Contrast volume during primary percutaneous coronary intervention and subsequent contrast-induced nephropathy and mortality. Ann. Intern. Med., 150, 170-177.

Marenzi, G., Bartorelli, A.L., Lauri, G., Assanelli, E., Grazi, M., Campodonico, J. \& Marana, I. (2003) Continuous venovenous hemofiltration for the treatment of contrastinduced acute renal failure after percutaneous coronary interventions. Catheter Cardiovasc. Interv., 58, 59-64.

Marenzi, G., Lauri, G., Assanelli, E., Campodonico, J., De Metrio, 
M., Marana, I., Grazi, M., Veglia, F. \& Bartorelli, AL. (2004) Contrast-Induced Nephropathy in Patients Undergoing Primary Angioplasty for Acute Myocardial Infarction. J. Am. Coll. Cardiol., 44, 1780-1785.

McCullough, P.A., Wolyn, R., Rocher, L.L., Levin, R.N. \& O’Neill, W.W. (1997) Acute renal failure after coronary intervention: incidence, risk factors, and relationship to mortality. Am. J. Med., 103, 368-375.

Mehran, R., Aymong, E.D., Nikolsky, E., Lasic, Z., Iakovou, I., Fahy, M., Mintz, G.S., Lansky, A.J., Moses, J.W., Stone, G.W., Leon, M.B. \& Dangas, G. (2004) A simple risk score for prediction of contrast-induced nephropathy after percutaneous coronary intervention: development and initial validation. $J$. Am. Coll. Cardiol., 44, 1393-1399.

Morcos, S.K., Thomsen, H.S. \& Webb, J.A.W.; members of contrast media safety committee of the European Society of Urogenital Radiology (ESUR) (1999) Contrast media induced nephrotoxicity: a consensus report. Eur. Radiol., 9, 1602-1613.

Mueller, C., Buerkle, G., Buettner, H.J., Petersen, J., Perruchoud, A.P., Eriksson, U., Marsch, S. \& Roskamm, H. (2002) Prevention of contrast media-associated nephropathy: randomized comparison of 2 hydration regimens in 1620 patients undergoing coronary angioplasty. Arch. Intern. Med., 162, 329-336.

Mueller, C., Buerkle, G., Perruchoud, A.P. \& Buettner, H.J. (2004) Female sex and risk of contrast nephropathy after percutaneous coronary intervention. Can. J. Cardiol., 20, 505-509.

Rihal, C.S., Textor, S.C., Grill, D.E., Berger, P.B., Ting, H.H., Best, P.J., Singh, M., Bell, M.R., Barsness, G.W., Mathew, V., Garratt, K.N. \& Holmes, D.R.Jr. (2002) Incidence and prognostic importance of acute renal failure after percutaneous coronary intervention. Circulation, 105, 2259-2264.

Senoo, T., Motohiro, M., Kamihata, H., Yamamoto, S., Isono, T., Manabe, K., Sakuma, T., Yoshida, S., Sutani, Y. \& Iwasaka, T. (2010) Contrast-induced nephropathy in patients undergoing emergency percutaneous coronary intervention for acute coronary syndrome. Am. J. Cardiol., 105, 624-628.
Shema, L., Ore, L., Geron, R. \& Kristal, B. (2009) Contrastinduced nephropathy among Israeli hospitalized patients: incidence, risk factors, length of stay and mortality. Isr. Med. Assoc. J., 11, 460-464.

Solomon, R., Werner, C., Mann, D., D’Elia, J. \& Silva, P. (1994) Effects of saline, mannitol, and furosemide to prevent acute decreases in renal function induced by radiocontrast agents. $N$. Engl. J. Med., 331, 1416-1420.

Toprak, O. \& Mueller, C. (2005) Differing definitions of contrastinduced nephropathy. Swiss Med. Wkly., 135, 630.

Valente, S., Lazzeri, C., Giglioli, C., Margheri, M., Comeglio, M., Nicolaci, L., Chechi, T. \& Gensini, G.F. (2006) Contrastinduced nephropathy in urgent coronary interventions. J. Cardiovasc. Med. (Hagerstown), 7, 737-741.

Van de Werf, F., Ardissino, D., Betriu, A., Cokkinos, D.V., Falk, E., Fox, K.A., Julian, D., Lengyel, M., Neumann, F.J., Ruzyllo, W., Thygesen, C., Underwood, S.R., Vahanian, A., Verheugt, F.W. \& Wijns, W.; Task Force on the Management of Acute Myocardial Infarction of the European Society of Cardiology. (2003) Management of acute myocardial infarction in patients presenting with ST-segment elevation. Eur. Heart J., 24, 28-66.

Weinstein, J.M., Heyman, S. \& Brezis, M. (1992) Potential deleterious effect of furosemide in radiocontrast nephropathy. Nephron, 62, 413-415.

Wickenbrock, I., Perings, C., Maagh, P., Quack, I., van Bracht, M., Prull, M.W., Plehn, G., Trappe, H.J. \& Meissner, A. (2009) Contrast medium induced nephropathy in patients undergoing percutaneous coronary intervention for acute coronary syndrome: differences in STEMI and NSTEMI. Clin. Res. Cardiol., 98, 765-772.

Wright, R.S., Reeder, G.S., Herzog, C.A., Albright, R.C., Williams, B.A., Dvorak, D.L., Miller, W.L., Murphy, J.G., Kopecky, S.L. \& Jaffe, A.S. (2002) Acute myocardial infarction and renal dysfunction: a high-risk combination. Ann. Intern. Med., 137, 563-570. 